\title{
Physiotherapeutic approach on the late phase of chikungunya: a case report
}

Aileciram Monialy Barros Marinho Ribeiro 1

Claudluce Marques Pimentel 2

Ana Clara Carvalho Gonçalves Guerra 3

Marcela Raquel de Oliveira Lima 4 1,3,4 Instituto de Medicina Integral Prof. Fernando Figueira (IMIP). Rua dos Coelhos, 300. Recife, PE, Brasil. CEP: 50.070-550. E-mail: marcelaraquelol@gmail.com
2 Faculdade Pernambucana de Saúde. Recife, PE, Brasil.

\begin{abstract}
Introduction: in April of 2016, the epidemiological bulletin reported 39,017 suspected cases of chikungunya fever in Brazil. The main symptoms of the disease in the acute phase are: high fever, polyarthralgia, back pain, headache and fatigue; while in the late phase present polyarthralgia or musculoskeletal pain which often has prolonged duration.

Description: in the late phase, the patient presented complaints mainly of: severe pain on the ankle joints wrists, elbows and shoulders. The medical handling consisted of continuous ultrasound sessions with the frequency of $1 \mathrm{MHz}$. The infrared laser with a dosage of $4 \mathrm{~J}$ and $3 \mathrm{~s}$ per point; TENS-burst with a pulse width of $250 \mathrm{us}$ and the frequency of $2 \mathrm{~Hz}$, carried out for 10 days. The physiotherapeutic treatment proposal was an innovation, however there are no medical literatures for completementary medication for the treatment which could reduce the use of analgesics and could cause prolonged comfort to the patient ratified by EVA and the $S F-36$.

Discussion: the use of ultrasound and low intensity laser are performed on the signs of chronic articular treatment in order to reduce inflammation, pain and joint stiffness. Therefore, a remarkable reduction of the algic state and the improvement of the patient's life along the $r$ ten consecutive sessions of a proposed protocol in this study, thus there is a need for further researches on this issue so it is possible to perform a medical handling based on evidences on the theme.
\end{abstract}

Key words Chikungunya fever, Arthralgia, Arbovirus infections 


\section{Introduction}

In the emerging and reemerging groups of infectious diseases, arbovirus transmitted by arthropods (mosquitoes) such as dengue (DENV) and chikungunya (CHIKV) are considered an important challenge to the public health. ${ }^{1}$ Among these viruses, the known type is the Alphavirus and the species in this type causes diseases which vary of mild fever to a severe polyarthritis and encephalitis. Chikungunya alpha virus (CHIKV) was originated from Africa and has caused recent global outbreaks associated to severe morbidity. The name "chikungunya" means "one that bends over" in Makonde language which is spoken in many regions in the Eastern Africa, due to the antalgic position which patients acquire during the period of the illness. 2

After the disease was isolated in 1952 in Tanzania, the first documented emergency regarding CHIKV occurred in the Southeast of Asia and in India settling in a sporadic cycle of urban transmission which continues until nowadays, which Aedes aegypti is the main vector. ${ }^{3}$ In October 2013, CHIKV arrived in America by the Caribbeans resulting in thousands of infections. In Brazil, the autochthonous transmission was detected in September 2014, in Oiapoque city(Amapá). During 2014, there were 2772 CHIKV cases confirmed and 6.784 cases were confirmed in 2015.4,5 According to the $13^{\text {th }}$ epidemiologic week bulletin $(01 / 03 / 2016$ to 04/02/2016) had notified 39.017 suspected cases of chikungunya fever in Brazil. The incidence rate of suspected cases was in 100,000 inhabitants, according to geographic regions, the Northeast area demonstrated the highest incidence rate of 56 cases 100,000 inhabitants. 6

Clinically, chikungunya fever incubation period is about 1-12 days, described as two phases, the acute phase in which symptomatic patients generally report an abrupt beginning of the disease characterized by high fever, polyarthralgia, back pain, headache and fatigue. These are clinical symptoms which appeared in 4-7 days, among the ones who had the disease declared that during the late phase presented arthralgia or musculoskeletal pain with the longest and the most frequent duration, lasting as long as weeks or months and sometimes, years. 7,8

Encountering this problematic scenario, we propose a physiotherapeutic approach which is still not mentioned in medical literature to relieve pain, swelling and immobility in the attempt of decrease the excessive use of anti- inflammatory in the late phase of chikungunya fever.

\section{Description}

I.M.S., 57 years old was addressed to the physical therapy service at the Rehabilitation Center and Physical Medicine Prof. Ruy Neves Baptista at the Instituto de Medicina Integral Prof. Fernando Figueira (IMIP), in Recife, Pernambuco, Brazil. The socioeconomic, demographic and clinical characteristics of the patient are described in Table 1.

The insect bite probably occurred on 10/30/2015, presenting pain, swelling, hyperemia and heat sensation on the joints of the body; itchy red spots on the skin, fever above 39 degrees sudden start and lasted five days. During the late phase the skin begins to peel, persistence of severe pain on the ankle, wrists, elbows and shoulders without significant improvement with the use of medication such as tramadol hydrochloride, dexamethasone, nimesulide, prednisone and among others, all of them with anti-inflammatory and analgesic effects.

The individual was evaluated in two moments in the rehabilitation service: in the introduction (5 months after the initial symptoms) and 10 days after. The analogic visual scale (EVA) was used in order to evaluate the intensity of the pain, $0=$ no pain and $10=$ the worst pain. Medical Outcomes Study $36-$ Item Short-Form Health Survey (SF-36) was used to evaluate the quality of life. This instrument is a multidimensional questionnaire composed of 36 items, grouped in eight topics functional capacity, physical aspects, pain, general health condition, vitality, social aspects, emotional aspects and mental health. Each topic presents a final score of 0 to 100 , zero (0) the worst condition and 100 the best health condition. The initial and final evaluations were accompanied by the same therapist. The patient neither had a previous physiotherapeutic treatment or cryotherapy.

Ethical Aspects: this case report was approved at the Ethics Research Committee under the number CAAE 56854216.3.0000.5201. The patient agreed to participate in this study and signed the informed consent form, according to the resolution 466 from the National Health Council.

After the patient was evaluated by a multidisciplinary team composed by a rheumatologist physician and some physical therapists $\mathrm{s} /$ he presented $\mathrm{EVA}=10$ on the shoulders and the elbows. Afterwards, with the physician and the patient's consent the medication treatment was suspended because the results were not what was expected. A proposal was made for a conservative treatment with a conventional physical therapist, which the initial purpose was to promote an anti-inflammatory and 
analgesics effect on the four most affected joints.

A protocol was planned and already applied starting in the first session by applying a continuous ultrasound with the frequency of $1 \mathrm{MHz}$, the intensity of $1 \mathrm{~W} / \mathrm{cm}^{2}$ for 6 minutes on the shoulders and 4 minutes on the elbows, followed by the infrared laser with a dosage of $4 \mathrm{~J}$ and $3 \mathrm{~s}$ per point having 5 points per joint, re-performing a total of $20 \mathrm{~J}$ and $12 \mathrm{~s}$ per joint and finalizing with the TENS-burst with a pulse width of $250 \mathrm{us}$ and the frequency of $2 \mathrm{~Hz}$, modulated by $150 \mathrm{~Hz}, 20$ minutes on each of the four joints distributing the electrodes in relation to the painful myotome.

This protocol was performed for during 10 days (Monday to Friday), where a progressive reduction of the algic state was observed daily. In relation to the beginning of EVA, there was a decrease of about two points at the end of each session which demonstrated stability in the reduced symptom. These EVA scores at the end of the sessions were similar to the following session, successively, with the exception of the shoulder joints in the second session. The elbow joints presented a good response and after the eighth session the beginning of EVA was equal to 0 maintaining as so until the end of the treatment (Figure 1).

The evaluation on the quality of life by SF-36 showed significant improvement on the post-intervention on the correspondent topics such as functional capacity, limitation due to physical aspects, emotional and social aspects, and vitality (Figure 1).

Table 1

Socioeconomic and clinical characteristics.

\begin{tabular}{ll}
\hline Gender & Female \\
Age (years) & 57 \\
Marital status & Single \\
Weight (Kg) & 85 \\
Height (cm) & 163 \\
Schooling & Elementary level up to 4th Grade \\
Monthly income & Less than one minimum salary \\
Occupation & Housewife \\
Physician diagnosis & Bilateral gonarthrosis and chikungunya \\
Physical Therapist diagnosis & Unspecific Arthralgia on the shoulders, elbows, wrists and cervical \\
Associated pathologies & HAS \\
Family pathologies & Not mentioned \\
Medication & Atenolol, losartan, hydrochlorothiazide \\
Consumes Alcohol & No \\
Smoker & No \\
Physical activities & No \\
History of falling down & Yes \\
AVD's restriction & Yes
\end{tabular}

AVD = daily life activities $\mathrm{HAS}=$ systematic hypertension blood pressure. 

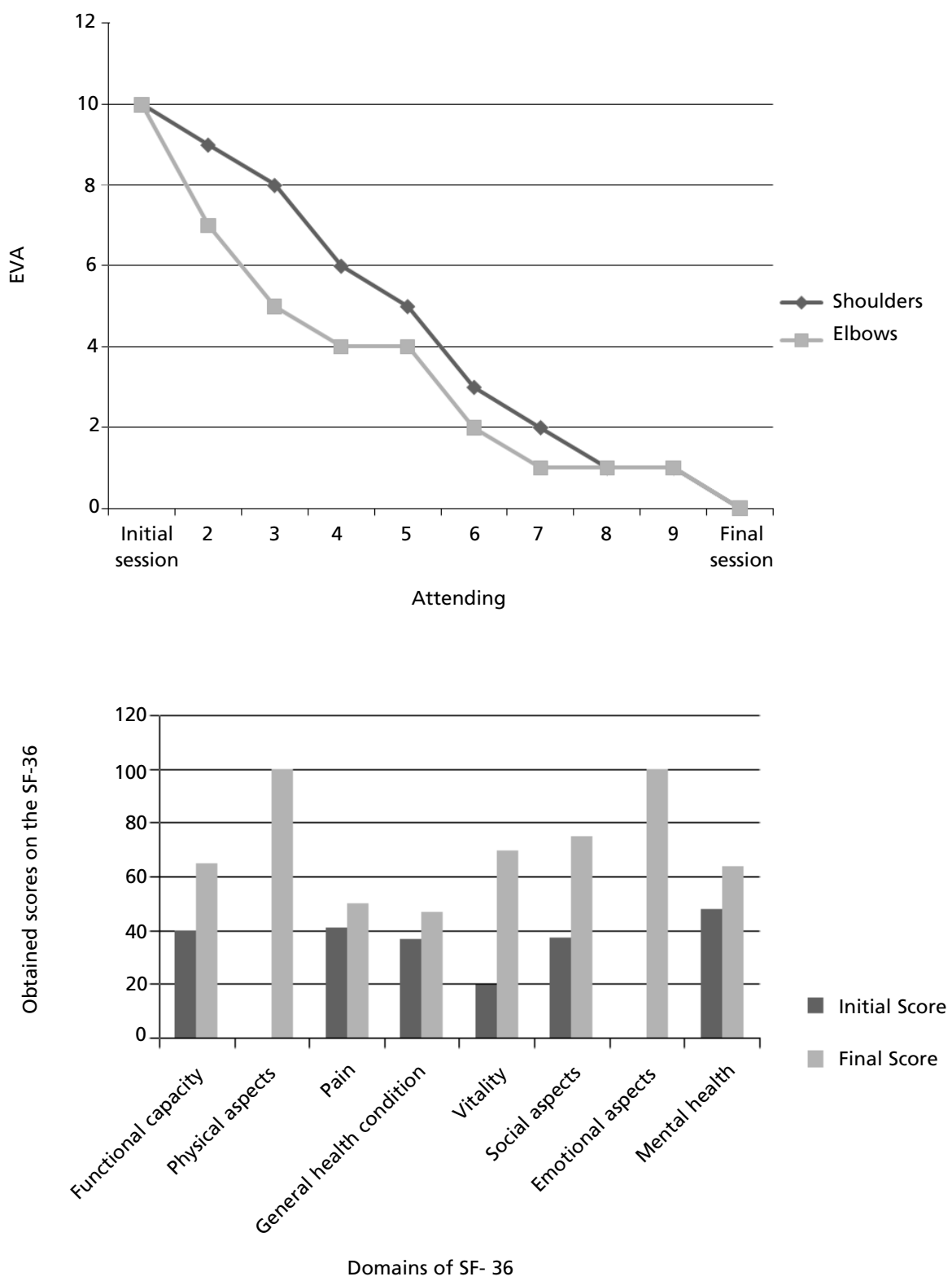

\section{Discussion}

The results in this present study showed significant improvement on the post-intervention to the quality of life evaluated by SF-36 and in the EVA scores possibly, as a consequence of the therapeutic modalities: continuous ultrasound which transmits heat by conversion, rising the blood flow by vasodilatation, the capillary permeability of muscle contraction speed the nerve conduction of the cell metabolism rate and collagen extensibility. TENS, an electric supplied transcutaneous which stimulates a large afferent sensorial fibers blocking the nociceptive primary fibers liberating endorphins and relieving the pain and the light by low power laser therapy which can produce photochemical reactions inside the cells which activates enzymes on the cellular level capable of increasing the mitochondrial function and the ATP (adenosine triphosphate - adenosia trifosfato)synthesis by increasing the cell proliferation and accelerating the healing process. 9

Historically, chikungunya has widespread in the 
world causing around 3 million infections per year. ${ }^{10}$ The epidemics in the Indic Ocean, Pacific Islands and the Americas continue to alternate the widespread of the disease by since the past years the outbreaks in the urban centers caused the virus to be spread by humans and mosquitoes, causing the global infection rates of chikungunya becoming variable. 1

The findings in current literatures corroborate with the symptoms described by the patient in this present study and was evidenced by EVA which presented maximum pre-intervention rate perhaps on the severe joint pain which the most important manifestation of CHIKV disease which occurs in most of the clinical cases.

Thiberville et al.11 in 2013 approached patients with rheumatic chronic manifestation such as rheumatoid arthriti (AR) spondylopathy and other types of non-classified rheumatisms as diagnosed and associated to chikungunya fever. Consequently, the conventional physical therapist adopted therapeutic resources used in the treatment for these rheumatic chronic manifestations in order to reduce inflammation, pain and joint stiffness.

This present study followed a protocol based on medical literature which applies the knowledge of heat transmission by ultrasound and other resources such as electrotherapy and low intensity laser used in the treatment of chronic rheumatic manifestation and which promotes the reduction of inflammation, pain and joint stiffness, as described by Beasley. 9

The existence of a possible inverse correlation between pain and the quality of life knowing that the

\section{References}

1. Gubler DJ. Human arbovirus infections worldwide. Ann NY Acad Sci. 2001; 951:13-24

2. Powers AM, Logue $\mathrm{CH}$. Changing patterns of Chikungunya virus: re-emergence of a zoonotic arbovirus. J General Virol. 2007; 88 (9): 2363-77.

3. Nasci RS. Movement of Chikungunya virus into the Western Hemisphere. Emerg Infect Dis. 2014;. 20 (8): 1394-5

4. Solignat M, Gay B, Higgs S, Briant L Devaux C. Replication cycle of chikungunya: a re-emerging arbovirus. Virol. 2009. 393 (2): 183-197.

5. Teixeira MG, Andrade AMS, Costa MCN, Castro JSM, Oliveira FLS, Goes CSB, Maia M, Santana EB, Nunes BT, Vasconcelos PF. East/Central/ South African genotype Chikungunya virus, Brazil, 2014. Emerg Infect Dis. 2015; 21: 906-7.

6. Brasil. Ministério da Saúde. Secretaria de Vigilância em Saúde. Boletim Epidemiológico. Monitoramento dos casos de dengue e febre de Chikungunya até semana epidemio- pain is among the main factor that can impact negatively the individual's quality of life for limiting the performance of the activities and leading to higher risks of stress and social isolation. 12

Some studies do not report conservative treatment performed by physical therapy for patients with chikungunya fever even if they are being treated by a disease with polyarthralgia of acute feverand in certain cases showing severe complications such as chronic arthritis. ${ }^{7}$ However, an improvement of a patient along the course of ten consecutive sessions with a protocol applied in this study although a study realized by Simon et al. ${ }^{13}$ disagreed with the analgesic effects of some physical therapist techniques on the persistent rheumatic manifestations such as ultrasound using hydrocortisone gel, hot/cold immersion therapy for the swelling and TENS for neuropathic pain.

Another negative aspect was the time for the therapy of one patient with the proposed treatment even though the patient complainted about the pain on many joints which led the session to be slower and the performance of other sessions for the other patients were not able to be performed at the same period.

This was one case only which is necessary for new researches to be performed with a greater and more significant samples regarding the issue to make it possible to associate among the applied therapeutic, the resolution of the case and prior inferences relating to the evidences on this topic.

lógica 13. 2016. [acesso em 15 mai 2016]. Disponível em http://portalsaude.saude.gov.br/images/pdf/2016/abril/27/2 016-014---Dengue-SE13-substitui----o.pdf

7. Thiberville SD, Moyena N, Dupuis-Maguiraga L, Nougairede A, Gould, EA, Roques P, Lamballerie X. Chikungunya fever: Epidemiology, clinical syndrome, pathogenesis and therapy. Antiviral Res. 2013; 99 (3): 34570

8. Chikungunya disease: gaps and opportunities in public health and research in the Americas. Wkly Epidemiol Rec. 2015; 90 (42): 571-6.

9. Beasley J. Osteoarthritis and Rheumatoid Arthritis: Conservative Therapeutic Management. J Hand Ther. 2012; 25 (2): 163-72.

10. Deller JJ, Jr \& Russell PK. An analysis of fevers of unknown origin in American soldiers in Vietnam. Ann Intern Med. 1967 (66): 1129-43.

11. Thiberville SD, Boisson V, Gaudart J, Simon F, Flahault A, Lamballerie X. Chikungunya Fever: A Clinical and 
Virological Investigation of Outpatients on Reunion Island, South-West Indian Ocean. PLoS Negl Trop Dis. 2013; 7 (1): e2004

12. Andrade FA, Pereira LV, Sousa FAEF. Mensuração da dor no idoso: uma revisão. Rev Latino-am Enfermagem. 2006; 14 (2): 271-6.

13. Simon F, Javelle E, Cabie A, Bouquillard E, Troisgros O, Gentile G, Leparc-Goffart I, Hoen B, Gandjbakhch F, ReneCorail P, Franco JM, Caumes E, Combe B, Poiraudeau S, Gane-Troplent F, Djossou F, Schaerverbeke T, CriquetHayot A, Carrere P, Malvy D, Gaillard P, Wendling D, Société de pathologie infectieuse de langue francaise. French guidelines for the management of chikungunya (acute and persistent presentations). November 2014. Med Mal Infect. 2015; 45 (7): 243-63.

Received on June 28, 2016

Final version presented on September 9, 2016

Approved on September 14, 2016 